\title{
A Reconsideration of the Classification of Two Types of CMEs
}

\author{
A.Q. Chen, C.T. Yeh, J.X. Cheng and P.F. Chen
}

Department of Astronomy, Nanjing University, Nanjing 210093, China

email: jdye@nju.edu.cn

\begin{abstract}
Conventionally coronal mass ejections (CMEs) are categorized into flare-associated and filament-associated types. Since there are also many CMEs of the overlapping type, we classify CMEs into three types in order to compare their characteristics. It is found that the three types of CMEs have quite similar distributions of apparent speeds, with small difference in the average speeds.
\end{abstract}

Keywords. Sun: coronal mass ejections (CMEs), filament, flares

\section{Introduction}

Conventionally coronal mass ejections (CMEs) are categorized into two distinct types, i.e., slow CMEs which are associated with filament eruptions and fast CMEs which are associated with solar flares (Sheeley et al. 1999). However, it has been noted that some CMEs are associated with both filament eruptions and solar flares (Feynman \& Ruzmaikin 2003). Therefore, it is worthwhile to reexamine the characteristics of CMEs of different types. In this paper, we divide CMEs into three types, i.e., (1) CMEs associated only with filament eruptions; (2) CMEs associated only with solar flares; (3) CMEs associated with both filament eruptions and solar flares, and investigate their characteristics in velocity distribution.

\section{Observations}

In this study, CME events observed by SOHO/LASCO (Brueckner et al. 1995) in 2002 are collected. The filament association is judged from the synthesis of the $\mathrm{H} \alpha$ images of Big Bear Solar Observatory, EUV images of SOHO/EIT (Delaboudiniere et al. 1995), and the core-like structures observed by LASCO. The judgment of the flare association is a little more complicated, which involves the backward extrapolation of the CME trajectory and some flare selection criteria. In this preliminary research, 257 out of the total 764 CMEs occurring in 2002 were selected into our sample, where 158 events belong to the first type, 77 events to the second type, and 22 events to the third type. The linearlyfitted apparent speeds of all the CMEs are presented in the CME catalog maintained by Gopalswamy et al. (http://lasco-www.nrl.navy.mil/cmelist.html).

\section{Data Analysis and Results}

The distributions of the three types of CMEs are shown in Figure 1. It is seen that the peaks of the three histograms do not differ significantly, though the tails at large speeds for the latter two types are enhanced compared to the filament-associated CMEs. The average speed of the three types is $476.6,532.4$ and $532.8 \mathrm{~km} \mathrm{~s}^{-1}$, respectively.

The relative portions of each type of CMEs at every $100 \mathrm{~km} \mathrm{~s}^{-1}$ interval are depicted in Figure 2, which indicates that relative distributions of the three types are rather 


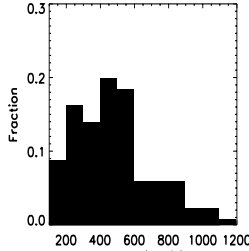
velocity $(\mathrm{km} / \mathrm{s})$

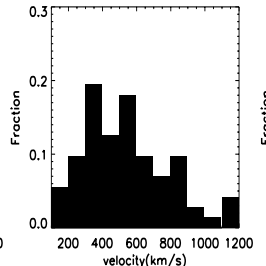

velocity $(\mathrm{km} / \mathrm{s})$

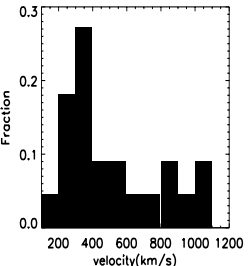

velocity $(\mathrm{km} / \mathrm{s})$

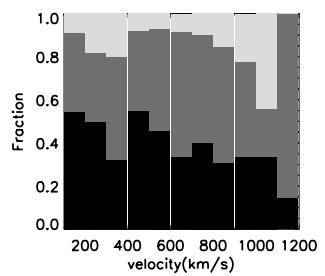

Figure 1. Histograms showing the relative distributions of velocity for the three types of CMEs. Left panel corresponds to events associated with filament eruptions only, middle panel to the events with solar flares only, and right panel to the events with both filament eruptions and solar flares.

Figure 2. Histogram showing the relative portions of the three types of CMEs at every $100 \mathrm{~km} \mathrm{~s}^{-1}$ velocity interval. Black columns correspond to the events associated with filament eruptions only, gray columns to the events with solar flares only, and white columns to the events with both filament eruptions and solar flares.

diffuse, though there is a slight trend that more events at high speeds belong to the flare-connected types.

\section{Discussion}

Phenomenologically it may not be appropriate to classify CMEs into two types, i.e., filament-associated slow CMEs and flare-associated fast CMEs. At least, there should be an overlapping type, which are associated with both filament eruptions and solar flares as in the classical CSHKP model. Therefore, we classify CMEs into three types, and investigate the distribution of apparent speeds for each type. It is found that the shapes of the distributions for the three types are quite similar, with somewhat stronger tails at small speeds and weaker tails at large speeds for the filament-associated type compared to the other two types. It is also noted that the average speeds for the three types of CMEs are not significantly different. Therefore, our results support the suggestion that there might not be intrinsic difference between various types of CMEs, and hence it is not physically significant to divide them into flare or filament associated. Their different velocity profiles, i.e., impulsive or acceleration types, are perhaps merely due to different time scale (Cliver 1999). In fact, during many CME events, which were previously thought not to be associated with flares, giant arcades or flares as weak as A-class are discerned (Wu et al. 2002; Zhang 2004).

\section{Acknowledgements}

The research is supported by FANEDD (200226), NSFC (10333040, 10221001 and 10403003) and NKBRSF (G20000784).

\section{References}

Brueckner, G.E., Howard, R.A., Koomen, M.J., et al. 1995, Sol Phys. 162, 357

Cliver, E.W. 1999, J. Geophys. Res. 104, 4743

Delaboudiniere, J.-P., Artzner, G.E., Brunaud, J., et al. 1995, Sol Phys. 162, 291

Feynman, J. \& Ruzmaikin, A. 2003, Sol Phys. 219, 301

Sheeley, N.R., Walter, J.H., Wang, Y.-W., \& Howard, R.A. 1999, J. Geophys. Res. 104, 24739

Wu, Y.Q., Tang, Y.H., Dai, Y., \& Wu, G.P. 2002, Sol Phys. 207, 159

Zhang, J. 2004, AAS. 204, 7204 